\title{
The changing landscape of genetic testing and its impact on clinical and laboratory services and research in Europe
}

\author{
Ros Hastings ${ }^{\star, 1}$, Guido de Wert ${ }^{2,3}$, Brian Fowler ${ }^{4}$, Michael Krawczak ${ }^{5}$, Eric Vermeulen ${ }^{3,6}$, Egbert Bakker ${ }^{7}$, \\ Pascal Borry $^{8}$, Wybo Dondorp ${ }^{2}$, Niels Nijsingh ${ }^{2}$, David Barton ${ }^{9}$, Jörg Schmidtke ${ }^{10}$, Carla G van El ${ }^{3,6}$, \\ Joris Vermeesch ${ }^{11}$, Yrrah Stol ${ }^{12}$, Heidi Carmen Howard ${ }^{13}$ and Martina C Cornel ${ }^{3,6}$
}

The arrival of new genetic technologies that allow efficient examination of the whole human genome (microarray, nextgeneration sequencing) will impact upon both laboratories (cytogenetic and molecular genetics in the first instance) and clinical/medical genetic services. The interpretation of analytical results in terms of their clinical relevance and the predicted health status poses a challenge to both laboratory and clinical geneticists, due to the wealth and complexity of the information obtained. There is a need to discuss how to best restructure the genetic services logistically and to determine the clinical utility of genetic testing so that patients can receive appropriate advice and genetic testing. To weigh up the questions and challenges of the new genetic technologies, the European Society of Human Genetics (ESHG) held a series of workshops on 10 June 2010 in Gothenburg. This was part of an ESHG satellite symposium on the 'Changing landscape of genetic testing', co-organized by the ESHG Genetic Services Quality and Public and Professional Policy Committees. The audience consisted of a mix of geneticists, ethicists, social scientists and lawyers. In this paper, we summarize the discussions during the workshops and present some of the identified ways forward to improve and adapt the genetic services so that patients receive accurate and relevant information. This paper covers ethics, clinical utility, primary care, genetic services and the blurring boundaries between healthcare and research.

European Journal of Human Genetics (2012) 20, 911-916; doi:10.1038/ejhg.2012.56; published online 28 March 2012

The application of the new genetic technologies that allow efficient examination of the whole human genome (microarray, next-generation sequencing (NGS)) will impact upon both laboratories (cytogenetic and molecular genetics in the first instance) and clinical/ medical genetic services. The high resolution of these technologies will help clinical geneticists to diagnose more cases than before. However, the implementation raises challenges that need to be addressed to safeguard a scientifically robust, ethically sound and socially acceptable level of service provision. The interpretation of the results in terms of their clinical relevance and the predicted health status poses a challenge due to the wealth and complexity of the information obtained. Clinicians need to decide how much information should be given to patients, (1) related to the symptoms they were referred for, (2) on other information of immediate health consequences, (3) on the carrier status of autosomal recessive conditions and (4) on variants of uncertain nature. Laboratory experts (and bioinformaticians) need to interpret the significance of the results for the clinician, and take responsibility for what they do or do not report. With the cytogenetic and molecular genetic service technologies merging closer, perhaps now is also the time to provide an integrated lab service.
Where incidental (ie, unsought or unexpected) findings concern currently unknown variants, common databases need to be developed to collect the evidence that becomes available. This evidence needs to be structured to form a suitable basis for evidence-based medicine. There will be an increased workload for diagnostic labs and an increased complexity of cases requiring interpretation. Moreover, clinical genetic services will need to expand or differentiate their services to cope with the increased complexity of cases and volume of work.

High-throughput technologies are rapidly changing the field of genetic screening. The ethical and policy challenges of large-scale applications in healthy individuals, for instance in newborn screening, need to be discussed. These new technologies also have the potential to detect every known genetic disease even before symptoms occur. Their responsible implementation has an impact on training, which should prepare health workers to decide and discuss when these technologies are or are not appropriate for certain medical indications. The relevance of the results in terms of sensitivity and predictive value needs to be evaluated. A central criterion to decide whether or not tests should be offered in regular health care is the

${ }^{1}$ CEQA and UK NEQAS for Clinical Cytogenetics, John Radcliffe Hospital, Oxford University Hospitals NHS Trust, Oxford, UK; ${ }^{2}$ Department of Health, Ethics and Society, Research Institute CAPHRI, Maastricht University, Maastricht, The Netherlands; ${ }^{3}$ Centre for Society and Genomics, Nijmegen, The Netherlands; ${ }^{4}$ ERNDIM, Metabolic Unit, University Children's Hospital Basel, Basel, Switzerland; ${ }^{5}$ Institute of Medical Informatics and Statistics, Christian-Albrechts University, Kiel, Germany; ${ }^{6}$ Clinical Genetics/EMGO Institute for Health and Care Research, VU University Medical Center, Amsterdam. The Netherlands; ${ }^{7}$ Deptartment of Human and Clinical Genetics, Leiden University Medical Centre, Leiden, The Netherlands; ${ }^{8}$ Centre for Biomedical Ethics and Law, KU Leuven, Leuven, Belgium; ${ }^{9}$ National Centre for Medical Genetics, Our Lady's Children Hospital, Dublin, Ireland; ${ }^{10}$ Institute of Human Genetics, Hannover, Germany; ${ }^{11}$ Center for Human Genetics, KU Leuven, Leuven, Belgium; ${ }^{12}$ Clinical Genetics, VU University Medical Centre, Amsterdam, The Netherlands; ${ }^{13}$ Institute of Biomedical Ethics, Basel, University of Basel, Switzerland

*Correspondence: Dr R Hastings, CEQA and UK NEQAS for Clinical Cytogenetics, Women's Centre, John Radcliffe Hospital, Oxford OX3 9DU, UK. Tel: +44 (0)1865 857644; Fax: +44 (0)1865 857632; Email: ros.hastings@ouh.nhs.uk

Received 10 October 2011; revised 28 February 2012; accepted 2 March 2012; published online 28 March 2012 
clinical utility, that is: whether the test will lead to an improved outcome. ${ }^{1}$ Guidelines for referral to clinical genetic services may need to be updated. Accreditation of services may need revision, or selfregulation could be appropriate.

It is important to know what the patient expects and/or needs. Who owns the whole-genome information and who decides what is relevant for the patient to know? Who decides on disclosure of genetic information to the patient-everything or selective? What are the risks of giving too much information, or not enough? What protection should there be for the patient? What should the healthcare system offer? The public at large must be informed on pros and cons of testing. And what will be the impact on clinical services of private companies offering genetic testing for multifactorial diseases through the internet? Biobanks and clinical services are merging closer. What does that mean for regulation, re-contacting and informed decision making?

To discuss the questions and challenges of new genetic technologies, the European Society of Human Genetics (ESHG) held a satellite symposium on the 'Changing landscape of genetic testing', coorganized by the ESHG Genetic Services Quality and Public and Professional Policy Committees and co-funded by the Netherlands Centre for Society and Genomics and Centre for Medical Systems Biology. The audience consisted of a mix of laboratory geneticists, clinical geneticists, ethicists, social scientists, patient organization representatives and lawyers.

In this paper, we summarize the discussions from the workshops and present some of the potential ways forward to improve and adapt the genetic services so that patients can receive accurate and relevant information. This paper covers ethics, clinical utility, primary care, genetic services and the blurring boundaries between healthcare and research.

\section{ETHICS: SEQUENCING VS ANALYSIS}

The NGS and whole-genome analysis (WGA) are rapidly becoming cheaper, making it affordable for clinical, research and screening purposes. Although it remains unclear what hopes are realistic, it is conceivable that we might attain the possibility of personalized medicine: prevention, diagnosis and treatment tailored to the individual's genome.

It is often assumed that NGS automatically results in WGA and that the two are more or less identical. The analysis performed after NGS may, however, still be targeted. Whether one performs a WGA instead of a targeted analysis (using filters to analyze only a selected part of the genome, such as all genes known to be related to a disease) is a choice that needs to be justified.

\section{DIAGNOSIS VS SCREENING}

When a clinically motivated WGA regularly produces results that have no direct relation to the initial problem and phenotype of the patient, and these results are communicated, is this part of the diagnostic practice, or is it a form of screening? Obviously, traditional demarcation lines between diagnostic testing and screening are under pressure-the distinction between these forms of testing seems to be blurring. It was suggested that diagnostics can be distinguished from screening by its starting point: a clinical problem and/or a phenotype. Diagnosis is centred around the attempt to locate the cause of a specific medical problem. WGA may also generate 'incidental findings'.

\section{INCIDENTAL FINDINGS, ADDITIONAL OR UNSOLICITED INFORMATION}

The terms 'unexpected' or 'incidental findings' are in themselves misleading, as it is a matter of statistics if and when a certain result will occur. It was suggested that perhaps the term 'additional information' or 'unsolicited information' is more appropriate.

In the diagnostic context, standard tests for particular clinical problems may produce additional results that were not sought. In discussing the implications of this phenomenon, it is important to keep in mind the distinction between targeted and WGA. The importance of using filters was acknowledged: a clinical test should address a particular clinical problem (eg, in case of a family history of breast cancer at a young age looking at breast cancer genes only; or one step further: not reporting mutations known to be nonpathogenic mutations). For some conditions, however, particularly unexplained intellectual disability, WGA is the only option as it is not possible to narrow down the scope of analysis by means of filters, therefore the analysis has to be non-targeted.

\section{Generic consent}

How do you involve the patient in the choice of whether and what unsolicited information should be disclosed? If a 'generic consent' approach is used, what sort of information should be supplied before asking a patient to consent to WGA? Can this information be satisfactorily framed into broader categories of possible outcomes? Would this approach satisfy the existing norms of consent? Obviously the availability of unsolicited information, that may physically and/or mentally harm the client-or his relatives, can lead to serious dilemmas particularly if a patient waives his right to know.

\section{Retention and recontacting}

What should be done with the huge amount of sequence data of a given patient, should it be stored for later use as required by current diagnostic guidelines (eg, future clinical questions or also for pharmacogenomics) or should one re-sequence the sample upon request? Clinical genetic centers may have most expertise, primary care physicians might perhaps not be the most obvious 'librarians' of patients' genomic information. An alternative could be to give the results to the patient, who then has discretion over what to do with them. Similarly to the storage of body tissues, there should always be a possibility to opt out of retention. Companies might offer to store and update information. Whether storage or re-sequencing is done depends on the cost of sequencing $v s$ the cost of storage. Data storage will require a legal framework to cover ethical questions, which might differ from country to country. When sequencing becomes cheaper, the majority of the participants supported the idea that in a clinical situation re-sequencing is the safest option.

Should the patient be recontacted when new or more information becomes available as a result of scientific progress, and if so, under what conditions? It would be both unsound and unpractical to propose a general duty to recontact, but at least within an existing treatment relationship, the doctor has a prima facie duty to inform his patient about findings which are relevant to him. But what is relevant? Many situations will, unfortunately, not be so clear-cut. An additional complication is when interests of third parties are at stake-here one may think of family members or of persons with a particular professional responsibility for the safety of others, such as air pilots.

\section{Newborn screening and prenatal uses}

It seemed clear to most participants that evidence is currently lacking for making WGA a sensible approach for newborn screening. The advantages are slim and there may be real disadvantages. International guidelines emphasize the child's right not to know, that is, their right to later decide about testing themselves. A related matter is the 
question as to whether parents should be prevented or discouraged from satisfying their 'curiosity' by means of direct-to-consumer (DTC) tests although a targeted analysis of a selection of disorders that pass the current criteria for screening would be wise.

In the case of prenatal testing, matters are even more complex. Collaboration between gynecologists and geneticists is essential. Interestingly, there was a slight difference between the 'American view' and the 'European view'. Whereas the first emphasizes the easy availability of new technologies and the choice of the individual pregnant woman, Europeans seemed more reluctant with regard to providing such choices without good reason. It was stressed that pregnancy is a time of great anxiety and that unclear results cause distress to women. Also, there were concerns of a possible information overload and the complexity of the choices under time constraints. In the workshop, the prenatal diagnosis discussions did not centre on the issue of abortion, instead it centred on the problems concerning the (future) child's right not to know. The resemblance between WGA prenatal testing and WGA in newborns indicates a blurring of the distinction between reproductive and non-reproductive screening.

The workshop emphasized the importance of clarity, validity, evidence-based practice and ethical reflection.

\section{CLINICAL UTILITY}

The question of how to build the evidence for the clinical utility of genetic tests exploiting high-throughput DNA sequencing technologies was discussed. The current standards for the implementation and provision of diagnostic services in health care are laid down, for example, in the ACCE framework, ${ }^{1}$ assessing analytic validity, clinical validity, clinical utility and ethical, legal and societal issues. New technologies could only change 'the landscape of genetic testing' if their clinical utility is clear. Clinical utility can scarcely be assessed in a purely academic setting, and for many rare diseases, clinical trials may not be feasible. The evidence must be built in clinical practice otherwise, sufficient data necessary to judge the impact upon the individual patient and their family made by the technology, and by the genetic variants newly detected with it, would often not be available.

At present, any reliable assessment of the clinical utility of new technologies for genetic testing is premature as the necessary data are not currently available. Moreover, before individual test results can be communicated to patients, the clinical validity of the data needs to be proven. Although it may be possible for a test to have clinical utility without having much clinical validity, this is rather unlikely to be the case for the (newly detected) genetic variants because, almost by definition, their clinical relevance will be unclear at the time they are first detected. Anyhow, in view of the apparent idiosyncrasies of the new sequence-based genetic tests, five key aspects should be considered before building up any evidence for their clinical validity or utility could be regarded a sensible option.

\section{Is building up the evidence worth the effort in the first place?} Media attention in combination with economic interests may well serve to generate a (perceived) demand for sequence-based genetic tests, even if such tests were met with scientific scepticism. ${ }^{2}$ Furthermore, although the predictive value of the (mostly incidental) genetic findings is currently unclear, the data may become scientifically useful in the future. On the other hand, if the effect size of newly detected variants that are sufficiently frequent to allow systematic follow-up is as low as suggested, for example, by recent genomewide association studies $\left(\mathrm{GWAS}^{3}\right)$, then their clinical evaluation will be both unproductive and unrewarding. Moreover, it remains to be seen whether the target population of sequence-based genetic tests, which will mostly be families with single-gene disorders, would be interested in participating in the extensive follow-up programmes required.

\section{Does building up the evidence seem feasible?}

In the present context, it appears mandatory that the quality and the level of detail of the phenotypes match the richness of the genotype data. This is because, contrary to common belief, reliable genotypephenotype relationships can only be established in retrospective manner if the phenotype is sufficiently specific for the genotype, and not vice versa. ${ }^{4}$ Moreover, the phenotype specificity of the genotype will likely vary between phenotypes (eg, a common disorder such as diabetes $v s$ diabetes at a young age in a family with an autosomal dominant inheritance pattern). Finally, as most of the required phenotypic data will have to come from clinical routine, their depth and quality will also be determined by the respective health care setting. Therefore, the feasibility of obtaining sufficient evidence for the clinical validity of a given genetic test will depend very much upon the test itself and on the clinical context in which it is provided.

\section{What is the advantage of building up the evidence in a clinical} context?

Based on the experience from GWAS of common disease, it appears likely that, in most instances, only rare genetic variants will have sufficiently strong phenotypic effects for them to warrant clinical follow-up. ${ }^{5}$ On the other hand, rarity implies that the clinical validity of diagnostic tests for such variants can only be assessed by studying the co-inheritance of genotypes and phenotypes in families. If it turns out that the quality of data obtained in a clinical context is insufficient and does not match the standards of common scientific practice, then additional efforts will have to be made, for example, through the establishment of systematic and standardized clinical assessment protocols.

\section{For which type of diseases will building up the evidence be primarily beneficial?}

Clinical validity and clinical utility can only be expected to be high for the genetic diagnoses of 'simple' single-gene disorders. Evidence for the clinical validity of newly detected, mostly rare genetic variants is likely to come from family studies, and for a variant to show familial aggregation, its penetrance must be high. As the initial target population of sequence-based tests will comprise families with single-gene disorders, the volume of accidentally detected genetic associations with common disease is likely to be rather small.

If the evidence were to be built up, who should drive the action? As any sensible assessment of clinical validity and utility will be confined initially to single-gene disorders, and then proceed in a clinical context, efforts in this direction would best be initiated and coordinated by disease-specific networks. The role of academia will depend on the extent to which research and health care infrastructures are intertwined. Concerted international efforts will be required for any endeavours to establish sequence-based tests as a means of routine genetic testing to be successful. Finally, individuals facing reproductive decision making may become 'captivated' by, and primed for such tests by commercial offers, including DTC carrier and prenatal diagnosis. ${ }^{6}$ 


\section{PRIMARY HEALTH CARE SERVICES}

Incorporating the available genomics knowledge into primary care has proven to be difficult. Clinical genetics services are adequately developed, but only few questions of well-informed patients on serious disorders reach these specialized centres. The introduction of whole-genome testing, may prove even more challenging for health care as a whole, and for primary care in particular. However, as almost any health problem nowadays has a genetic component, reflection is needed on possibilities, prerequisites and barriers for incorporating genetics/genomics for general practice, occupational medicine and public health.

How far do we want to go in assessing and preventing risk? Should the genomes of the whole population be sequenced? Or could determining relevant biomarkers at different moments in life be more useful?

\section{What is necessary for incorporation of NGS in primary care?}

Currently, many general practitioners have difficulties in adequately stratifying genetic risk and knowing when to refer patients. Also, knowledge is lacking to address questions from patients based on DTC genetic testing, although rarely encountered. The European Medicines Agency could be an important stakeholder on the European level if, similar to the USA Food and Drug Administration, it could decide whether a test is analytically and clinically valid. In the United Kingdom, the National Health Service identifies useful and cost-effective applications. GPs' time and knowledge constraints might be met by recent developments, such as the creation of 'primary care GP super clinics', as planned in the Australia, by embedding a genetic counselor in primary care. Genetics needs to be 'normalized' in both the general public and (primary) care; fears and myths need to be addressed. Apart from different professional (eg, clinical chemists, clinical geneticists and medical specialists) and health care bodies and government agencies (eg, the EU Directorate General for Health and Consumer Affairs), the general public is important for risk assessment aiming to prevent disease. Needs and wishes of public and the primary care professionals must be met. Whereas patient organizations have an established role as stakeholder, there is no equivalent format for 'healthy' people beside perhaps consumer organizations.

\section{The role of stakeholders and organizations}

Realistically speaking genetic knowledge is applied only when it is integrated in guidelines and financed by health insurance. Primary care could pilot projects to determine how genetics can be incorporated in the field. More is expected from supranational organizations, such as the provision of guidelines and the establishment of registries to create clarity about genetics applications in primary care. It is important to stimulate dialog and collaboration between geneticists, different professional societies and public health organizations, especially regarding population screening. Bridges need to be built because early specialization in university curricula often hinders communication between these different professional domains. In the case of screening programs, evaluation should be in place to ensure the population is not harmed. For DTC testing, post-marketing surveillance should be organized, preferably by independent institutions and/or researchers. As commercial parties are here to stay, collaboration may be better than exclusion. Self-regulation is preferred to legal guidance because national and supranational laws are outdated by the time they finally become legal code. Laws also cannot prevent genetic practices that work across borders, such as commercial testing.

\section{GENETIC SERVICES}

Routine genetic diagnostics is starting to use NGS and it will generate data/information not necessarily related to the indication but could be used for other health care purposes (screening, diagnosis and personalized medicine). Expertise teams for medical genomics or clinical geneticists could upon request discuss the 'screening' results and the wider implications of the results for the patient and give health advice based on the genome data generated. This will bring a substantial change in the scope for clinical geneticists and will need training programs for professionals and public. For research projects, one would need more specific consent forms and an agreement about the data usage and storage (anonymous or coded).

The challenges of NGS from a laboratory perspective will be the identification of new syndromes/genetic diseases as well as 'normal variants' lacking clinical significance. This will require that all genetic laboratories interpret the clinical significance of the information obtained.

\section{How can we best restructure laboratory services and determine and interpret data/information that should be made available to clinicians?}

A core analytical facility is envisaged that would service the various genetic disciplines. This facility should guarantee the maintenance of best-quality standards given the present analytical limitations and the built-in error rate using external quality control. Mechanisms should be installed to exclude mix up of samples. In this vision, the local speciality laboratory (molecular/cytogenetics/biochemical genetics) might undertake confirmatory and repeat testing if needed, select information from the NGS data according to the clinical question, and arrange or perform additional tests as and when needed. In order to fulfill this function, there is need for freely available patient databases/comprehensive data on all known mutations and detailed documentation of clinical disease and non-disease associations. Massive bioinformatics systems and optimal interaction between the laboratory and the clinician are crucial.

\section{Should all genetic referrals be offered NGS or are there exceptions} with a targeted approach, for example, prenatal testing?

As long as targeted testing is better and cheaper, this is preferred, however, in the future NGS is a realistic scenario. It was not considered appropriate for prenatal diagnosis. It could be that NGS is eventually very cost effective and easy to perform and may provide helpful information about confounder traits in monogenetic disorders that this technology becomes the first and main option. The analysis performed after NGS may, however, be targeted using filters.

It was considered that, in general, genetic reports should be restricted to the clinical question but there may well be certain specific disease-related genotypes that should be disclosed in the interest of the patient, but clear consensus guidelines will be needed for this. There might be different disclosure levels related to the clinical presentation, potential outcome changes (treatment) and particular patient-related factors. There is also a need to protect laboratory workers in those situations where a potential dilemma regarding disclosure exists, for example, when lab results reveal an incestuous relationship.

Amalgamation of the cytogenetics and molecular genetic disciplines into genomic services was broadly supported, integrated with proteomics and metabolomics services. Also pharmacogenetics fits within this structure. Specialist training remains needed given the expected massive increase of the scientific and technical knowledge base. A generic component of training seems inevitable especially for molecular and 
cytogenetics (as has been started in the UK and The Netherlands). New disciplines will be needed, for example, genome informatics.

\section{BLURRING OF BOUNDARIES BETWEEN RESEARCH AND CLINICAL GENETIC TESTING SERVICES}

The goal of research is the generation of new evidence and knowledge, and that of clinical care to endorse patients' best interests. Although in theory this distinction may appear clear, in practice the division between both contexts is in fact ambiguous. ${ }^{7,8}$ For example, it is not always easy for patients to distinguish genetic tests that are offered as a part of their clinical management from tests that are offered as a part of a research protocol. ${ }^{9,10}$ Studies also show that some researchers are likely to be seen, and see their own research activities, as a way of providing care. ${ }^{11}$ Finally, DTC genetic testing blurs many boundaries; between consumers and patients as well as between consumers and research subjects.

Previous research has highlighted the various concerns that accompany the blurring of boundaries between research and genetic testing services. ${ }^{8,12}$ Is the informed consent procedure adequate? Do patients have a sufficient understanding of what is happening to the sample they provide and what the potential implications are as a consequence of their participation in the research study? Given this understanding, or lack thereof, is their participation voluntary? Moreover, could pressure arise when the question to participate in research is being asked by a treating healthcare professional. Interestingly, it was also reported that some research activities are re-classified as non-research activities in order to avoid the requirements linked to ethical approval of their research. ${ }^{12}$ During the workshop, two different case scenarios were discussed.

\section{Case 1: DTC genetic testing companies}

The first scenario made reference to a particular DTC genetic testing company, which offers whole-genome testing. Consumers can order various genetic testing packages through the company's websites (ie, for genealogy purposes, health purposes or both). By signing the company's consent form for the genetic testing services, consumers also agree that their genetic and other contributed personal information will be stored in the company's research databases, and authorized company personnel will conduct research using said databases. Is blurring between a consumer purchasing the genetic test and being part of research taking place in this context? Workshop participants voiced concerns about a blurring between health promotion and health care. Although the information provided might make people reflect over genetic and environmental factors that influence their health, it does not meet the standards of clinical validity and utility and companies usually have disclaimers stating that their services are not intended to be diagnostic services. That being said, companies also make many health-related claims on their websites and it has been documented that some consumers do not understand this. ${ }^{13}$ Furthermore, consumers may overvalue the predictive character of the information they receive. Consequently, discussion arose about the difference between information provision and interpretation. Providing a huge amount of information will be increasingly possible in genetics, but workshop participants wondered who will interpret the data and how will this be done?

\section{Informed consent}

There is a lack of differentiation between the informed consent procedure for the purchasing of a genetic test and for the research that is performed using consumers' data. Consumers might not be sufficiently informed about the (particular forms of) research activities to make an informed choice. For example, it was documented that some research participants would not want to participate in studies of homosexuality. Many may have problems with the commercial practices that could follow from the research activities. Through the research activities of DTC companies, consumers are becoming research participants and are, in fact, paying to participate in research.

\section{Privacy and confidentiality}

Confidentiality and anonymity have become important standards in genetic research. However, this might be challenged more and more in the future, for instance when consumers/research participants of DTC companies share their results with others through online communities. Some genetic research, although anonymised, might create group harm in certain communities.

\section{Case 2: longitudinal multigeneration research}

The second case scenario is a Dutch research cohort study (http:// www.lifelines.net): a triple generation longitudinal population-based study among 165000 inhabitants of the northern Netherlands to study genetic and environmental risk factors for prevalent multifactorial disorders. All research participants receive questionnaires and a basic medical examination. The computerized patient files of the GPs are the main source for follow-up. The research study provides the GPs of research participants with the results of the medical tests. Again anonymity is at stake: is a guarantee of privacy still possible when data are collected from three generations in a certain delimited geographical region? Return of the results needs to be discussed: should that go further than the basic medical examinations?

The blurring of boundaries between research and genetic testing services generates a need for more policy development on issues concerning anonymity, privacy, confidentiality, ownership, return of results and informed consent in both the research and clinical realms.

\section{CONCLUSIONS}

The workshop identified the following priorities for further policy development:

- Professionals from laboratory and clinic as well as patients, ethicists and social scientists need to collaborate to develop policy in the changing landscape of genetic testing.

- Techniques investigating the whole genome will generate additional findings: 'always expect the unexpected'. Before starting to use these techniques a policy on 'unsolicited findings' must be defined.

- When sequencing becomes cheaper the re-sequencing of a patients DNA, as opposed to storing the original data, is likely to be the safest option provided the latter is not a requirement by guidelines.

- Generic consent should be obtained for WGA/NGS.

- Generic training programmes for laboratory and clinical staff (including GPs) will need to evolve that incorporate these new technologies as disciplines merge.

- A core analytical facility is envisaged that would service the various genetic disciplines.

- Ethical, legal and social issues must be addressed in tandem with scientific and technological progress.

\footnotetext{
1 Zimmern RL, Kroese M: The evaluation of genetic tests. J Public Health 2007; 29: 246-250.

2 Weiss KM: Tilting at Quixotic trait loci (QTL): an evolutionary perspective on genetic causation. Genetics 2008; 179: 1741-1756.
} 
3 Eichler EE, Flint J, Gibson G et al: Missing heritability and strategies for finding the underlying causes of complex disease. Nat Rev Genet 2010; 11: 446-450.

4 Weiss KM, Terwilliger JD: How many diseases does it take to map a gene with SNPs? Nat Genet 2000; 26: 151-157.

5 Goldstein DB: Common genetic variation and human traits. New Engl J Med 2009; 360: 1696-1698.

6 Waalen J, Beutler E: Genetic screening for low-penetrance variants in protein-coding genes. Annu Rev Genomics Hum Genet 2009; 10: 431-450.

7 Cooke S, Crawford G, Parker M, Lucassen A, Hallowell N: Recall of participation in research projects in cancer genetics: some implications for research ethics. Clin Ethics 2008; 3: 180-184.

8 Easter MM, Henderson GE, Davis AM, Churchill LR, King NM: The many meanings of care in clinical research. Sociol Health IIIn 2006; 28: 695-712.
9 Hallowell N, Cooke S, Crawford G, Lucassen A, Parker M: Distinguishing research from clinical care in cancer genetics: theoretical justifications and practical strategies. Soc Sci Med 2009; 68: 2010-2017.

10 Parker M, Ashcroft R, Wilkie AOM, Kent A: Ethical review of research into rare genetic disorders. BMJ 2004; 329: 288-289.

11 Ponder M, Statham H, Hallowell N, Moon JA, Richards M, Raymond FL: Genetic research on rare familial disorders: consent and the blurred boundaries between clinical service and research. J Med Ethics 2008; 34: 690-694.

12 Yentis SM, Dawson AJ: Medical studies with 'no material ethical issues'-an unhelpful, confusing and potentially unethical suggestion. Clin Ethics 2006; 1: 234-236.

13 McGuire A, Diaz CM, Wang T, Hilsenbeck S: Social networkers' attitudes toward directto-consumer personal genome testing. Am J Bioethics 2009; 9: 3-10. 